\title{
NIIF Y MIPYMES: RETOS DE LA CONTABILIDAD PARA EL CONTEXTO Y LA PRODUCTIVIDAD*
}

\author{
Mauricio Gómez Villegas**
}

* doi: 10.11144/Javeriana.cao29-53.nmrc. Este artículo es producto de la Línea de investigación en teoría y regulación contable, financiada por la Universidad Nacional de Colombia desde agosto de 2013 hasta diciembre de 2015. Grupo de Investigación Contabilidad, Organizaciones y Medioambiente. El artículo se recibió el 0807-2016 y se aprobó el 10-10-2016. Una versión previa de este artículo fue publicada en los documentos de trabajo de la Escuela de Administración y Contaduría Pública, Facultad de Ciencias Económicas, Universidad Nacional de Colombia: http://www.fcenew.unal.edu.co/publicaciones/images/doc/documentos-administracion-25.pdf. Sugerencia de citación: Gómez-Villegas, M. (2016). NIIF y MIPYMES: retos de la contabilidad para el contexto y la productividad. Cuadernos de Administración, 29 (53), 49-76. http://dx.doi.org/10.11144/ Javeriana.cao29-53.nmrc.

* * Doctor en Contabilidad de la Universitat de València, España. Profesor asociado y director de Innovar Journal, Escuela de Administración y Contaduría Pública, Facultad de Ciencias Económicas de la Universidad Nacional de Colombia, Bogotá, Colombia.

Correo electrónico:mgomezv@unal.edu.co 


\section{NIIF y MIPYMES: retos de la contabilidad para el contexto y la productividad}

\section{ResUmen}

Las micro, pequeñas y medianas empresas (MIPYMES) constituyen la mayoría de organizaciones en el tejido empresarial mundial. Dado el proceso de estandarización contable internacional liderado por el IASB, surge la NIIF-PYMES, esta norma asume el enfoque de las NIIF para empresas cotizadas. Dado que las MIPYMES no participan en mercados de valores este artículo examina si la regulación contable del IASB puede promover la preparación de información contable y financiera útil para la toma de decisiones y la productividad en las MIPYMES. El artículo caracteriza el enfoque y las limitaciones que impone la regulación contable internacional, para la información de estas empresas, enfatizando en los retos para que sea útil en la toma de decisiones y para la productividad.

Palabras clave: NIIF para PYMES, regulación contable, productividad, mipymes, financiarización.

Clasificación JEL: M41, P10, M20

IFRS and MSMEs: accounting challenges for context and productivity

\author{
NIIF e MPMEs: desafios \\ da contabilidade para \\ o contexto e para a \\ produtividade
}

\section{ABstract}

Micro, small and medium enterprises (MSMEs) constitute most of organizations within the global entrepreneurial network. When the international accounting standardization process led by IASB is established, the IFRS-MSMEs comes into existence, a regulation that assumes the focus of IFRS for listed companies. Since the MSMEs do not participate in stock markets, this article examines whether accounting regulation of IASB can promote the preparation of useful accounting and financial information for decision-making and productivity within the MSMEs. The article characterizes the focus and limitations that the international accounting regulation imposes for the information of these enterprises, emphasizing its challenges so that it becomes useful for decision-making and productivity.

Key words: IFRS for MSMEs, accounting regulations, productivity, MSMEs, financialization.

JEL Classification: M41, P10, M20

\section{Resumo}

As micro, pequenas e médias empresas (MPMEs) constituem a maioria das organizações no contexto empresarial mundial. Tendo em vista o processo de padronização contábil internacional liderado pelo Conselho de Normas Internacionais de Contabilidade (IASB), surge a NIIF-MPMEs. Essa norma assume a abordagem das NIIF para empresas cotizadas. Considerando que as MPMEs não participam de mercados de valores, este artigo examina se a regulamentação contábil do IASB pode promover a preparação de informação contável e financeira útil para a tomada de decisões e para a produtividade nas MPMEs. Além disso, caracteriza a abordagem e as limitações que a regulamentação contábil internacional impõe para a informação dessas empresas, dando ênfase aos desafios para que seja útil na tomada de decisões e para a produtividade.

Palavras-chave: NIIF para MPMEs, regulamentação contábil, produtividade, MPMEs, financeirização.

Classificação JEL: M41, P10, M20 


\section{Introducción}

El proceso de regulación contable internacional que se viene gestando desde inicios del presente siglo ha tenido implicaciones sustanciales. El International Accounting Standards Board-IASB- (Consejo de Normas Internacionales de Contabilidad) se ha convertido en el regulador global de la información contable y financiera. Con la emisión del International Financial Reporting Standard -IFRS- (Norma Internacional de Información Financiera, NIIF), para Pequeñas y Medianas Entidades -PYMES-, el alcance de sus pronunciamientos pretende extenderse a múltiples formas organizacionales. La NIIF para PYMES, de la que ya se han emitido dos versiones, implica que el modelo contable del IASB abarca múltiples organizaciones (entidades) y no sólo empresas o grupos económicos que cotizan en bolsa. Pese a ello, el enfoque explícito del modelo contable planteado por el IASB da prevalencia a la producción de información para inversores, acreedores y prestamistas, actuales y potenciales, que actúan en mercados financieros. Como consecuencia, la maximización del valor para el accionista y la valoración de la firma son propósitos implícitos en la lógica de los tratamientos contables prescritos por estos estándares (Watts y Zuo, 2016). Dado que mayoritariamente las micro, pequeñas y medianas empresas no cotizan en bolsa y enfrentan condiciones meso, macro y microeconómicas diferenciadas en cada contexto, resulta relevante preguntarse: ¿puede la regulación contable del IASB promover la preparación de información contable y financiera útil para la toma de decisiones y la productividad en las MIPYMES?

Para abordar este cuestionamiento, este artículo plantea una caracterización del enfoque, las limitaciones y los retos que impone la actual regulación contable internacional para la contabilidad de las Micro, Pequeñas y Medianas empresas. En el análisis de este trabajo se incorporan las microempresas porque ellas son el origen y la base estructural de evolución hacia las PYMES. Asimismo, los requerimientos de contabilidad simplificada para las microempresas, en algunas jurisdicciones, han buscado la convergencia con la NIIF de PYMES. Se enfatiza en el contexto empresarial latinoamericano, aunque se presentan datos y estadísticas de fuentes secundarias en un contexto internacional más amplio.

El enfoque metodológico del trabajo es normativo y crítico (Ryan, Scapens, Theobald, 2002); normativo pues se plantean argumentos sobre el "deber ser" de la regulación dirigida a las micro, pequeñas y medianas empresas; crítico porque articula dudas sistemáticas y racionales sobre los planteamientos oficiales o de la autoridad. Asimismo, se plantean reflexiones desde referentes teóricos y evidencias empíricas relativas al contexto y operación de este tipo de empresas. Se realizó una revisión de la literatura 
internacional más representativa sobre el tema, lo que permitió seleccionar teorías institucionales en economía, sociología y contabilidad para explicar y comprender la dinámica de las MIPYMES.

El artículo se estructura en cinco apartados, además de esta introducción. En la primera sección se caracteriza el proceso de globalización contemporáneo y la tendencia de transformación de la economía mundial hacia la financiarización. En el segundo apartado se presenta el origen, el enfoque y la estructura de la regulación contable a nivel internacional; concretamente se describe el marco en el que se posiciona el IASB y la estructura de la regulación que ha emitido. La tercera parte del trabajo se enfoca en caracterizar el contexto internacional de las MIPYMES y se presenta la necesidad de su abordaje desde un enfoque "glocal". En el cuarto apartado se realiza un análisis general de la NIIF para PYMES, resaltando algunos de los problemas y de los retos de una regulación contable para la toma de decisiones y la productividad, lo que reclama la comprensión del contexto de las MIPYMES. Finalmente, en el quinto apartado se presentan unas breves conclusiones y las implicaciones del trabajo en la investigación futura.

\section{Globalización y transformación de la economía mundial: Financiarización}

Desde finales de la década de 1970 se viene gestando un proceso de transformación de la economía, de las relaciones entre el Estado y las corporaciones empresariales, de integración de los mercados financieros a escala global y, por todo ello, de modificación en las relaciones sociales de generación y distribución del valor (Vercellone, 2009). Tanto la revolución de las tecnologías de la información y la comunicación -TIC- como el proceso de desregulación e innovación en los mercados financieros, están en la base de estos cambios (Pérez, 2005).

\subsection{Características de la globalización financiera}

Para comprender el contexto de surgimiento y posicionamiento del modelo contable internacional promovido por el IASB, a continuación se describen cuatro procesos característicos de la globalización financiera.

La primera característica distintiva del entorno económico actual es la expansión e interconexión de los mercados financieros (de capitales y de intermediación). Esto se ha 
dado gracias a la promoción de sus virtudes como mecanismos de asignación "eficientes" de recursos para la inversión empresarial, para la gestión del riesgo financiero y hasta para la financiación de los Estados (Pérez, 2005). La política pública y el rol activo de las instituciones multilaterales, tanto de origen estatal como no gubernamental han sido claves en este proceso, llámense FMI, BM, BID, G7, G20, entre otros.

La desregulación de los mercados consiste en el lento desmonte de la soberanía nacional en la determinación de los patrones, los requerimientos y los límites para la acción de los agentes en los mercados financieros. No obstante, los mercados requieren reglas para operar y su expansión se acompañó del surgimiento y posicionamiento de organismos reguladores privados de escala global que plantean defender el interés público, pero que estructuran el campo de acción en los mercados de conformidad con objetivos concretos, no necesariamente consensuados o basados en intereses de los ciudadanos o las naciones (Bezemer, 2010; Arnold, 2012).

La expansión de los mercados financieros implica que los individuos (por medio de la bancarización, la suscripción de contratos de seguro o los esquemas pensionales, entre otros), las empresas (por la emisión de títulos de propiedad y de deuda, así como por la suscripción de instrumentos de cobertura de riesgos) y el Estado (por la ausencia de emisión monetaria, por la privatización de empresas estatales, por la emisión de títulos públicos de deuda para cubrir el déficit fiscal, etc.) participen de forma activa en los mercados financieros y dependan de su dinámica, intermediarios y resultados.

En este contexto emergen y se consolidan grandes organizaciones que congregan recursos financieros de fuentes muy diversas, bien por la prestación de servicios especializados, bien por ser medios para canalizar recursos de inversión financiera especializada: los fondos institucionales de inversión (Pérez, 2005; Çelik e Isaksson, 2014). La consolidación y preponderancia de tales agentes constituye la segunda característica de la globalización financiera. Los inversores institucionales buscan administrar de forma especializada los recursos de diversas actividades: las pensiones de la sociedad, la gestión especializada del aseguramiento en salud para los ciudadanos, o la constitución de fondos de cobertura (Hedge Fund), entre otros (Orléan, 2006). La reforma de la Administración Pública fue determinante para trasladar una gran cantidad de las funciones, otrora estatales, hacia agentes privados, consolidando diversos "mercados". Allí los fondos institucionales se convirtieron en el canal para llevar los flujos de recursos que tales actividades generan hacia los mercados financieros (Bezemer, 2010). 
La dinámica y los procesos de operación de los fondos institucionales de inversión reta la concepción tradicional de "inversores" (Young, 2006). Aquí los agentes que administran los fondos ( $p$. ej. de pensiones) son expertos financieros que gobiernan recursos del público y que no operan según los parámetros de los inversores convencionales, anónimos y desprovistos de información o de poder, que se enfocan en la gestión del retorno-riesgo de sus "ahorros". Los gerentes de los fondos de inversión aprovechan la ventaja de gestionar grandes recursos, fuera del control de los propietarios de los mismos (que son simplemente afiliados, en gran medida por obligación de Ley, antes que a motu proprio) (Orléan, 2006). En general, los afiliados a los fondos de pensiones o a las administradoras financieras de salud no están interesados ni tienen la capacidad de gobernar las instituciones que administran sus pensiones, generando una libertad particular en la acción de estos jugadores. Estas instituciones se configuran como los nuevos inversores en la globalización financiera, que tienen la capacidad de poner en jaque las políticas públicas de un país, bajo la idea según la cual “los mercados no ven con buenos ojos este o tal gobierno o esta política pública" (Bezemer, 2010). Lo mismo ocurre a nivel de las empresas que reciben financiación de tales inversores institucionales.

Una tercera característica determinante del momento actual de la globalización consiste en la hegemonía cultural de las finanzas. El filósofo italiano Antonio Gramsci definió la Hegemonía Cultural como los modos de pensar, las orientaciones teóricas y los modos de conocer, dirigidos por las clases o grupos dominantes, que configuran y transforman la estructura económica y política de la sociedad. Esto significa que la hegemonía cultural se refiere al surgimiento de cuerpos conceptuales, teorías y mecanismos de comprensión de la realidad, que logran transformar nuestra forma de ver y de actuar en el mundo (Gruppi, 1978).

Por consiguiente, la hegemonía cultural de las finanzas se refiere al proceso según el cual las finanzas se posicionan como la matriz de conceptos, como el cuerpo teórico por excelencia, para comprender, intervenir y actuar en la sociedad (Power, 2010; Orléan, 2006). Esta hegemonía tiende a transformar la estructura social de tal manera que busca convertirse en el lenguaje del sentido común. De allí que todos los días, en los telediarios, en los periódicos, en la radio, se provea información, datos y análisis del comportamiento de los mercados financieros. Implica que se argumente que todo depende de lo que ocurra en los mercados (locales e internacionales). No importa, ni es necesario por supuesto, que todos los ciudadanos sepan, conozcan, los conceptos, las teorías o los mecanismos financieros. En el nivel de los expertos, las finanzas se convierten en el lugar privilegiado para conseguir legitimidad (Power, 2010). De lo que se trata es que la cotidianidad de 
los sujetos esté atravesada por su lenguaje, por sus formas de ver, nombrar, operar y de interpretar el mundo. Por supuesto, ocurriendo esto, la estructura social se "construye" y "dirige" en tal dirección.

Una cuarta y última característica que debemos resaltar de la globalización contemporánea, es la transformación de la concepción de la empresa. Debido a los cambios tecnológicos y sociales, la empresa comienza a ser vista y a operar como una red de contratos, antes que como un proceso transformativo centralizado. La entrada en la producción mundial de los países emergentes del sudeste asiático, particularmente de China e India, proveyó de un volumen de mano de obra sin igual a la economía global. Junto con la revolución de las TIC y las políticas para profundizar e integrar el comercio mundial, todo llevó a una transformación en la concepción y operación de la empresa. La tercerización y externalización de los procesos productivos implica que la actividad transformadora se traslade, espacial y organizacionalmente, fuera de las fronteras de empresa y, en muchas ocasiones, de los países donde opera o tiene sus mercados (Pérez, 2005).

El proceso de tercerización y externalización ha implicado la construcción de una red de intermediarios necesarios para integrar lo que ha sido separado o diseccionado buscando una reducción de los costos de producción. En concreto, si una empresa de vehículos automotores encarga la producción de las partes electrónicas a terceros en China, los motores en Corea, la silletería en Italia, los chasises en el sur de Francia y ensambla en Alemania, el conjunto de procesos necesarios para integrar el vehículo automotor dará lugar a una nueva economía donde lo vital son los servicios necesarios para la integración. Lo que se observa, por tanto, es que la empresa de automotores, dueña de la marca, gestiona el diseño, la comercialización, el marketing y el proceso de investigación y desarrollo, en concreto: lo estratégico. Por ello, concentra la mayor parte del precio final, como porción de los "servicios" que ella misma gestiona. Los vehículos se siguen "produciendo", pero la producción como transformación material se ve desvalorizada en el ciclo económico, puesto que se paga menos por ese trabajo que es trasladado a maquilas, en los países en vías de desarrollo y, cada vez más, en algunos enclaves dentro de los antiguos países industrializados (Pérez, 2005; Coriat et al., 2012). De esta forma, las actividades socialmente mejor valoradas permanecen en las oficinas de las corporaciones y países centrales donde la compañía "agrega valor a la firma".

En síntesis, ahora hablamos de firma, antes que de empresa y menos de fábrica. La firma es un espacio especializado en gestionar una red de contratos que externaliza costos y riesgos de la producción y busca "concentrar" el valor en los servicios medulares o 
estratégicos. Esta concentración se hace de muchas maneras, entre otras, gestionando operaciones en los mercados financieros (Coriat et al., 2012).

\subsection{El gobierno de la empresa y la financiarización}

Es necesario reiterar que las características antes descritas no son condiciones suficientes para explicar la globalización actual. No obstante, sí son condiciones necesarias para su comprensión, especialmente para identificar las implicaciones de estos cambios y su relación con la contabilidad. Estas características buscan darnos una idea más general de la actual etapa financiarizada de la globalización. La financiarización de la empresa implica un cambio en su modelo de gobierno (Aglietta y Reberioux, 2009; Archel y Gómez, 2014).

La empresa es la célula constitutiva de la formación social del capitalismo. Es la unidad de producción y el centro de la articulación de las relaciones sociales para intercambiar bienes y servicios: particularmente el trabajo humano. Desde un punto de vista técnico, la división y coordinación del trabajo dentro de la empresa es el objeto de la administración o la gestión. Desde la dimensión social y política, la empresa ha sido por años el centro de generación y distribución del valor. Por lo tanto, la articulación de las relaciones técnicas de producción y sociales de distribución dentro de la empresa, implican la existencia de múltiples modelos para su gobierno. El gobierno de la empresa es también dependiente de las variadas formas de capitalismo que subsisten a nivel mundial (Albert, 1992; Dufour, 2009).

La conjugación de las transformaciones que se han narrado previamente, lleva a un nuevo gobierno de las empresas. En síntesis, el gobierno de la empresa, su corporate governance, se constituye por cuatro elementos: i) los objetivos empresariales, ii) la forma como se divide y coordina el trabajo, iii) la manera como se integran los procesos productivos y, iv) los mecanismos en los que se distribuye el valor generado en la producción (Tirol, 2001; Coriat et al., 2012).

En el capitalismo gerencial (managerial) que se configuró durante el siglo XX, tanto en Europa como en Estados Unidos, y que luego fue importado en el proyecto desarrollista a nivel mundial, el objetivo de la empresa se dirige hacia la búsqueda de la eficiencia productiva para incrementar el resultado empresarial (Aglietta y Reberioux, 2009). La administración científica y la producción dirigida desde la mirada de la ingeniería buscó la satisfacción de necesidades, articulando la capacidad empresarial para producir de manera más "eficiente" (Archel y Gómez, 2014). La experticia técnica en la actividad misma 
dirigía los procesos de división y coordinación del trabajo. El foco en la productividad reclamó la integración vertical y horizontal para generar economías de escala. Se buscó la integración tanto de unidades productivas como de procesos y personas (trabajadores). La fábrica es la forma prototípica de articulación para que la división e integración del trabajo permitiera generar rendimientos crecientes a escala. Las actividades de financiación en la empresa pretendían la consecución de recursos frescos para aumentar la capacidad productiva (la inversión en capital físico y en tecnología) y con ello la actividad generadora de beneficios fruto de la transformación y del desarrollo del objeto productivo. El valor, entonces, se genera y distribuye dentro de la empresa. La visión taylorista según la cual se debe retribuir a los trabajadores en función de la productividad, implica una forma de distribución del valor generado, para ampliar la posibilidad de consumo generando vínculos y procesos de mayor integración económica (Coriat et al., 2012).

Con los cambios gestados con la globalización financiera se transforma el gobierno de la empresa (Dufour, 2009). El objetivo empresarial pasa a ser la maximización del valor para el accionista: la búsqueda del rendimiento financiero. El rendimiento financiero se puede obtener sin producción directa en la que se apliquen los recursos, pues es la retribución del capital por su circulación o simple disponibilidad. La empresa comienza a ser gestionada desde la experticia financiera, desplazando a la lógica productiva (Aglietta y Reberioux, 2009). Ahora la manera de aumentar la rentabilidad y disminuir los costos implica la externalización y la tercerización. La firma externaliza grandes partes de sus procesos productivos y se concentra en actividades o servicios que generan mayor rendimiento financiero. La reingeniería, la subcontratación y el desarrollo de mecanismos de competencia interna (el proceso de desalarización, la contratación por proyectos y la medición del desempeño por objetivos), transforman la dinámica de la división y la coordinación del trabajo. Las actividades financieras ya no se preocupan tanto por conseguir más o mejores activos para producir, sino que se enfocan en la gestión de la financiación para conseguir rentabilidad, bien con el apalancamiento (los pasivos), bien con las operaciones de portafolio (inversiones financieras) (Archel y Gómez, 2014). Desde esta mirada, aparentemente, el autointerés y el énfasis en la retribución financiera son suficientes para explicar la división y la coordinación del trabajo, con lo que la empresa se convierte en la firma o ficción jurídica-contractual definida por Jensen y Meckling (1976).

En esta nueva forma de gobierno de la empresa, para el accionista, la doctrina central de la gestión es la maximización del valor. El valor, ahora, se genera y distribuye (concentra) en los mercados financieros. De esta manera, la empresa se transforma y se convierte en un engranaje vital del proceso de financiarización. “Financiarización significa que, en 
el proceso económico, la lógica financiera se sobrepone a la lógica productiva" (Giraldo, 2005). Esto es una transformación en el régimen de acumulación. No significa que ya no se produzca, sino que la producción, en sí misma, ya no es el centro social de la actividad económica más valorada. La forma social de juzgar la economía y de retribuir los esfuerzos económicos cambia. Se transforman los valores sociales (en un sentido axiológico) para que cambien los valores económico-financieros. En el plano microeconómico “al nivel de la empresa, la financiarización se refiere a la dominación de la maximización del valor para los accionistas como el objetivo corporativo por encima de todo" (Guttman, 2009, p. 22). La riqueza se sigue produciendo en la transformación concreta (ahora en maquilas, p. ej., en las que se producen los teléfonos i-Phone), pero el valor ahora es generado en una cadena ampliada y es distribuido (o concentrado) en los mercados financieros y en las bolsas (donde el valor de mercado de Apple, define quien es el dueño de la riqueza generada). Por ello es determinante distinguir la riqueza de su valor financiero, hipertrofiado por la financiarización (Arnold, 2012; Muller, 2014).

\section{Origen, estructura y enfoque de la regulación contable a nivel internacional}

Quizás no sea una simple coincidencia que el proceso de armonización internacional en materia contable iniciase de manera decidida en 1973, con la creación del International Accounting Standards Committee -IASC- unos años después del proceso de ruptura de los pactos internacionales de paridad en el patrón oro para las divisas (tratado de Bretton Woods, roto en 1971) y cuando se daban los primeros pasos en la desregulación de algunos mercados financieros a nivel internacional. La creación del IASC fue promovida por las entidades de normalización y regulación contable de nueve países pioneros en el comercio internacional en la época (Australia, Canadá, Francia, Alemania, Japón, México, Países Bajos, el Reino Unido y Estados Unidos). Desde la época de su creación y hasta el año 2001, el IASC emitió 41 Normas Internacionales de Contabilidad. Un organismo anexo, el Standards Interpretation Committee, produjo otro tanto de pronunciamientos bajo la figura de interpretaciones.

\subsection{La nueva arquitectura financiera internacional}

Con las crisis desatadas a nivel internacional por los diversos procesos de desregulación e integración global de los mercados financieros (que durante los años 1980 y 1990 se hicieron más recurrentes y devastadoras), se inicia un proceso de evaluación de las causas y determinantes de tal inestabilidad financiera global. Durante más de una década 
se diagnostica la fragilidad de la arquitectura financiera internacional, luego del estudio del conjunto de instituciones, normas y prácticas que viabilizan la integración y la operación de los mercados financieros a nivel global. Fruto del diagnóstico se establece la urgencia de transformar este entramado institucional, promoviendo la adopción de diferentes patrones, reglas o estándares que permitan la prevención, la identificación, la gestión y la superación de las crisis financieras (Eichengreen, 2000). Por ello, la Nueva Arquitectura Financiera Internacional es un conjunto de estándares que busca influir en el comportamiento macroeconómico de los Estados, en las operaciones de los Bancos y las Entidades Financieras y en la gestión, la divulgación de información y en el control de las empresas. La Tabla 1 esquematiza los principales estándares vinculados con la nueva arquitectura financiera internacional.

\section{Tabla 1}

Estándares de la nueva arquitectura financiera internacional

\begin{tabular}{|c|c|c|}
\hline & Tipo de estándar & Sector de aplicación \\
\hline 1 & Divulgación de datos macroeconómicos & \multirow{3}{*}{$\begin{array}{l}\text { Estándares aplicables al estado/ } \\
\text { gobierno }\end{array}$} \\
\hline 2 & Prácticas de transparencia fiscal & \\
\hline 3 & Transparencia de las políticas monetaria y fiscal & \\
\hline 4 & Supervisión bancaria & \multirow{4}{*}{$\begin{array}{l}\text { Estándares aplicables al sector } \\
\text { financiero y banca }\end{array}$} \\
\hline 5 & Supervisión de los seguros & \\
\hline 6 & Normatividad bursátil & \\
\hline 7 & Sistemas de pago & \\
\hline 8 & Gobierno o gestión de la empresa (Corporate Governance) & \multirow{3}{*}{ Estándares aplicables a las empresas } \\
\hline 9 & Contabilidad & \\
\hline 10 & Auditoría & \\
\hline 11 & Régimen de insolvencia y derechos de los acreedores & Todos los sectores \\
\hline
\end{tabular}

Fuente: construcción propia.

\subsection{Surgimiento del IASB y estructura de su regulación}

El International Accounting Standards Board -IASB- surge de la transformación del IASC, frente al proceso de construcción de una Nueva Arquitectura Financiera Internacional. El cambio implicó reformas en la constitución de los órganos de financiación, en la composición del Consejo, en los mecanismos de su gobernanza, en el ajuste de los procesos de emisión normativa y en la profundización de la lógica financiera en la regulación contable. En el fondo, cada vez más el regulador contable está constituido no solo por miembros 
de la profesión contable, puesto que se incorporan permanentemente observadores y otros interesados. No obstante, existen poderosos grupos de interés con capacidad de lobby y seguramente, con interés y capacidad para la captura del regulador (Bengtsson, 2011; Deegan y Unerman, 2011).

El cambio de emisión de las Normas Internacionales de Contabilidad -NIC- (IAS por sus siglas en inglés) a Normas Internacionales de Información Financiera (IFRS por sus siglas en inglés), ocurrido desde 2001, no es simplemente de denominación; implica la transición de la regulación de la contabilidad, concebida como proceso, a concentrarse en los informes financieros, entendidos como productos del proceso. En términos generales, podría decirse que la contabilidad como proceso se circunscribe a las dinámicas organizacionales, pero los informes deben ser homogéneos, para garantizar, según los propósitos del IASB, la transparencia y la comparabilidad en los mercados financieros. Por ello se plantea que la regulación del IASB no se fundamenta en reglas concretas, sino en principios, que deben ser interpretados por los preparadores de la información financiera en el contexto de cada modelo de negocio.

Diferentes y paulatinos "consensos" fueron posicionando al IASB como el regulador de más aceptación en este ámbito. El hecho más significativo fue la decisión de la Unión Europea, en 2002, según la cual los grupos empresariales cotizantes en bolsas europeas debían observar las normas del IASB en la formulación y presentación de sus estados financieros consolidados al cierre de 2005. También en 2002, el acuerdo de Norwalk implicó un proceso de legitimación del IASB, que buscó aproximar el marco normativo internacional con las normas emitidas por el Financial Accounting Standards Board -FASB- órgano de normalización de la contabilidad en Estados Unidos. Este acuerdo, que significó que el IASB asimilara la lógica de los USGAAP, no llegó a buen puerto, puesto que viene siendo lentamente abandonado desde 2012 y aún subsisten diferencias profundas entre los dos marcos normativos.

Desde el año 2001 y hasta noviembre de 2016, el IASB ha emitido 16 Normas Internacionales de Información Financiera (NIIF). Asimismo, fue promulgada la NIIF para Pequeñas y Medianas Entidades, que ya cuenta con dos versiones: 2009 y 2015. Por su parte, el órgano de interpretaciones correspondiente ha emitido los International Financial Repoting Interpretation Committee -IFRIC-. También continúan vigentes 25 Normas Internacionales de Contabilidad, NIC (emitidas por el IASC) y 5 de sus interpretaciones (emitidas por el Standard Interpretation Committee -SIC-) que fueron suscritas y aprobadas por el IASB. 
La regulación, en teoría, está soportada en un Marco Conceptual, que fue originalmente emitido en 1989, modificado fruto del trabajo conjunto con el FASB en 2010 y que está en proceso de ajuste luego de la ruptura de los acuerdos de Norwalk ${ }^{1}$; el nuevo marco es liderado de forma independiente por el IASB desde $2013^{2}$. Un borrador de este documento fue puesto en discusión en 2015 y espera emitirse formalmente en 2017 (IASB, s.f.).

\subsection{Propósito de la información financiera y enfoque del modelo IASB}

El Marco Conceptual del IASB establece claramente que:

El objetivo de la información financiera con propósito general es proporcionar información financiera sobre la entidad que informa que sea útil a los inversores, prestamistas y otros acreedores existentes y potenciales para tomar decisiones sobre el suministro de recursos a la entidad. Esas decisiones conllevan comprar, vender o mantener patrimonio e instrumentos de deuda y proporcionar o liquidar préstamos y otras formas de crédito. (IASB, 2010; 0B2)

Esta elección de satisfacer las necesidades de información de los inversores (Staubus, 1961), es la característica fundamental de lo que en contabilidad conocemos como el "paradigma de la utilidad de la información". Aunque la información financiera debería ser útil para múltiples usuarios y propósitos, en el enfoque del IASB se busca satisfacer a los proveedores de capital que actúan en mercados financieros.

Este enfoque de toma de decisiones tiene impactos sobre los criterios concretos de reconocimiento, medición, valoración y mantenimiento de capital; en síntesis, constituyen un modelo contable concreto. Bajo este enfoque, los activos son aquellos bienes o derechos susceptibles de generar flujos futuros de efectivo, con independencia de si son o no propiedad del ente. Asimismo, la valoración de los activos y los pasivos, debe propender por incorporar datos del mercado, cuando existan, o desarrollar valuaciones con base en modelos. Al mismo tiempo, la incorporación de los nuevos instrumentos financieros (derivados, forwards y opciones) en la información contable, se vuelve determinante para el modelo contable (Biondi, 2011).

1 http://www.fasb.org/jsp/FASB/FASBContent_C/ProjectUpdatePage\&cid=900000011090

2 http://www.ifrs.org/Current-Projects/IASB-Projects/Conceptual-Framework/Pages/Conceptual-Framework-Summary.aspx 
Por todo lo anterior, un concepto central del enfoque regulatorio es el Fair Value, que "es el precio que sería recibido por vender un activo o pagado por transferir un pasivo en una transacción ordenada entre participantes del mercado en la fecha de la medición". (IFRS 13, Párrafo 9; IASB, 2015a)

La aplicación del valor razonable busca, sobre todo, que la información contable relativa al valor de la empresa se aproxime al valor bursátil de los títulos que representan la propiedad de las compañías cotizadas. Por años, los financieros y otros grupos de interés se han quejado de la diferencia entre el valor contable y el valor bursátil de las empresas (Biondi, 2011). Estas diferencias ponen en tela de juicio las valoraciones y los precios en los mercados. Por ello, la preocupación de analistas y expertos financieros ha sido cómo aproximamos la información contable a los precios de mercado. La respuesta contemporánea llega con la regulación contable del IASB (Chua y Taylor, 2008; Muller, 2014). En otra época se señaló que la diferencia entre el valor bursátil y el contable se explicaba por los intangibles de las empresas tecnológicas. Con la crisis de las empresas punto com ese argumento fue desvirtuado en gran medida.

Este modelo termina incorporando la volatilidad de los precios (muchas veces determinados por comportamientos oportunistas, irracionales o especulativos) a la información contable de las empresas. La información financiera, así concebida, es un instrumento central en la operacionalización del cambio de gobierno de la empresa, ahora bajo la influencia directa de los mercados financieros y de capitales (Dufour, 2009; Biondi, 2011; Muller, 2014). La información financiera de una empresa viabiliza el proceso de concentración de valor (cuando no de expropiación del mismo) en los mercados financieros. Ya no importa representar las transacciones o la situación económico-financiera desde el punto de vista productivo (Bezemer, 2010). Lo importante, ahora es que represente la potencialidad de los beneficios futuros que justifican la transferencia hoy del valor (y de parte de la riqueza) para los actores financieros. Es el reconocimiento de valorizaciones e ingresos potenciales no realizados, con base en el optimismo desbordado de los gerentes, presionados por las demandas de los inversores y los "mercados".

\section{Las MIPYMES: el contexto y la producción "glocal"}

Las micro, pequeñas y medianas empresas -MIPYMES- constituyen el mayor volumen de organizaciones productivas en todos los países del mundo. Su importancia en la generación de empleo, en el proceso económico o cadena de valor de las grandes empresas y 
su creciente importancia en el PIB mundial, son sin duda elementos determinantes que llaman la atención de gestores públicos y de la investigación académica, también en el ámbito contable (Pratten, 1998; Collis y Jarvis, 2002).

\subsection{Una aproximación a las MYPIMES}

Sólo para presentar una panorámica general, el informe del Banco Mundial y el Grupo Dalberg, titulado "Report on Support to SMEs in Developing Countries Through Financial Intermediaries" (World Bank y Dalberg Grup, 2011), señala que en los países emergentes, el número de microempresas muy pequeñas formalmente establecidas se encuentra entre 25 y 30 millones, las microempresas serían entre 55 y 70 millones, y existirían, según las estimaciones del estudio, entre 285 y 340 millones de empresas no formalizadas y con un solo empleado. Según el informe, las microempresas muy pequeñas son negocios unipersonales formalizados.

Desde el punto de vista de la producción, contamos con algunos datos y estimaciones para contrastar la participación de las MIPYMES. Según el estudio reseñado (World Bank y Dalberg Group, 2011), en los países de bajos ingresos el 63\% del PIB es generado por la economía informal y el sector de MIPYMES. Del total del PIB, un 16\% sería fruto de microempresas y pymes formales, mientras el $47 \%$ del PIB sería fruto de la economía informal. Por el contrario, en las economías de altos ingresos, el 51\% del PIB sería generado por el sector de las MIPYMES, mientras que solo el 13\% sería fruto de la economía informal.

Existen algunos criterios para caracterizar las MIPYMES, bien por el monto de sus activos, patrimonio, nivel de ventas o por el número de sus empleados. Aun con estos criterios, las diferencias en términos de magnitud son significativas. El umbral de definición por volumen de activos entre empresas de la misma región latinoamericana no permite una comparación adecuada y puede llevar a conclusiones ambiguas.

Por lo anterior, las MIPYMES no constituyen un grupo homogéneo que pueda ser caracterizado de manera general en el ámbito global, simplemente por variables financieras de su operación o estructura interna. Estas empresas comparten problemas como la ausencia de estructura formal, débil o nula financiación y bajas competencias técnicas (World Bank y Balberg Group, 2011). Más importante aún es que las MIPYES a escala internacional se diferencian por las condiciones contextuales en que surgen y operan. Es decir, por variables meso, macro y microeconómicas que conviene evaluar. 


\subsection{El contexto: condiciones meso, macro y microeconómicas}

La meso-economía se refiere a la dimensión estructural de los procesos económicos. La estructura económica en la que operan las MIPYMES difiere de manera sustantiva. No es igual la forma de operación de dos microempresas, si una de ellas está situada en Colombia o México, que si operara en Alemania. La estructura económica hace que la disponibilidad de bienes y servicios públicos, tales como infraestructura portuaria, vial, de movilidad y conectividad nacional, tenga implicaciones, genere costos de transacción y externalidades (tanto positivas como negativas). Ni que hablar del sistema legal y de la administración pública. En este mismo sentido, las condiciones logísticas a las que se enfrenta un mediano empresario tienen diferencias contextuales sustantivas de orden meso-económico. La capacitación, la formación y las condiciones estructurales de vida de los trabajadores de uno u otro contexto, tendrán implicaciones directas sobre la productividad en una u otra MIPYME. La formalización empresarial es también una característica determinante de los condicionantes meso-económicos de las MIPYMES.

De acuerdo con el informe titulado "Perspectivas económicas de América Latina 2013", de la OCDE y la CEPAL, la productividad de las medianas empresas italianas, francesas, alemanas y españolas, promediaba el $80 \%$, en función de la productividad de las grandes empresas, mientras que las medianas empresas de Argentina, Brasil y Chile alcanzó en promedio menos del $50 \%$ de la productividad de las grandes empresas de tales países. En cuanto a las Microempresas, el caso de Alemania y Francia es excepcional, representando una productividad promedio cercana al $75 \%$ de la lograda en las grandes empresas, mientras que, en Perú, Brasil y Chile, la productividad está por debajo del $10 \%$ sobre la misma base de comparación (OCDE y CEPAL, 2013).

Desde el punto de vista macroeconómico la tasa de cambio, el tipo de interés, el nivel de desempleo, entre otras, son variables determinantes de la operación y los procesos productivos de las MIPYMES. A inicios de mayo de 2016, la tasa de interés fijada por el Banco Central de Brasil llegó al 14,25\%; en la misma fecha, la tasa de interés del Banco Central de la Federación Rusa, era del 11\%; la reserva federal de los Estados Unidos tenía una tasa de interés, en el mismo momento, de 0,50\% (Investing.com, s.f.). Estas tasas se convierten en punto de referencia para las tasas de interés comercial en cada país. Las MIPYMES requieren financiación bancaria como fuente determinante para su operación y, como queda planteado, no es lo mismo para una empresa endeudarse en Estados Unidos o Brasil que hacerlo en Colombia. 
También, el costo de la mano de obra estará muy relacionado con los niveles de desempleo, entre muchas otras variables. Según el informe del World Bank y Dalberg Group (2011), en los países de bajos ingresos, el 78\% del empleo es generado por las MIPYMES, mientras que en los países de ingresos medio-altos estas mismas empresas generan el $59 \%$ del empleo.

Desde el punto de vista microeconómico, la situación también implica diferencias significativas. La forma como las MIPYMES se articulan a los procesos productivos resulta determinante: mientras que en el contexto italiano, los encadenamientos productivos del cuero implican la cooperación entre pequeñas empresas, en el contexto de la cadena de valor de las curtiembres en Colombia, las MIPYMES compiten y terminan expuestas a retribuciones anodinas, bien por los grandes compradores-productores de manufacturas, o bien por los precios del mercado internacional de baja calidad impactando en los precios pagados por el consumidor final.

Según el informe "Integrating Developing Countries SMEs into Global Value Chains" de UNCTAD (2010), las MIPYMES coreanas se articulan en los eslabones de producción y de desarrollo tecnológico medio, mientras que en las PYMES latinoamericanas aún prevalece la focalización en manufactura y ensamble. El caso de México es paradigmático con los encadenamientos productivos en algunas regiones en la industria del automóvil, particularmente con Wolkswagen, que también incluían etapas de contenido tecnológico (aunque medio y bajo) (UNCTAD, 2010). En este contexto, muchas de las labores de maquila, por ejemplo, en la manufactura de textiles, están entregadas a MIPYMES con baja articulación y que compiten entre ellas en el campo de la producción de prendas de vestir a muy bajos costos, no solo en Asia sino en Centro y Suramérica (UNCTAD, 2010).

Por todo lo anterior, las variables meso, macro y microeconómicas no deben obviarse al momento de acometer el estudio de las MIPYMES, de las prioridades y de las restricciones que enfrentan y de las necesidades de información contable y financiera que se articulan con tales prioridades, restricciones y formas de operación.

\subsection{La necesidad de un enfoque "glocal" para la productividad y la información contable contextual}

En consonancia con lo antes señalado, planteamos que una evaluación estratégica del rol de la contabilidad para la productividad y el contexto, en el caso de las MIPYMES, 
reclama un enfoque "Glocal”. La comprensión de la situación de estas empresas nos reta a pensar que:

Aunque, por varias razones, sigamos usando el concepto de globalización, sería preferible reemplazarlo para determinados fines por el de glocalización. Este último concepto tiene la ventaja de tomar en cuenta tanto las cuestiones espaciales como las temporales. Y al mismo tiempo, el énfasis sobre la condición global -esto es, en la globalidad- nos empuja a que nuestro análisis e interpretación del mundo contemporáneo sean a la vez espacial y temporal, geográfico e histórico. (Robertson, 2003, p. 269)

\section{Críticas y retos de la regulación contable en el contexto de las MIPYMES}

Como se ha señalado, la situación general de las MIPYMES reta a los científicos sociales, a los gerentes y a los formuladores de políticas públicas. En este apartado se presentan algunos de los retos que enfrenta la contabilidad de estas empresas. Para ello, se evalúan críticamente algunos de los tratamientos prescritos por la NIIF de PYMES y, desde la teoría de la contabilidad, se plantea la necesidad de una regulación contable sintonizada con la productividad y la representación del contexto.

\subsection{Crítica al enfoque}

La NIIF de PYMES fue desarrollada bajo la prioridad de simplificar los tratamientos y requerimientos exigidos en las NIIF plenas. Es decir, se asumió que las PYMES requieren menos información que las grandes empresas. Quizás otra interpretación sobre esta línea de acción sería señalar que se consideró que estas empresas no están en capacidad de producir la misma información por el grado de complejidad técnica de los tratamientos. Ambas posiciones podrían ser incorrectas. Las PYMES no requieren menos información contable y financiera que las grandes empresas: requieren una información distinta. Por supuesto, exigir a las PYMES información para satisfacer necesidades de inversores en mercados financieros, es poner una carga de costos de información, sin beneficios reales. De allí que la segunda justificación también sería errónea. Exigir un tratamiento más sencillo a las PYMES, que está también dirigido a producir información para los mercados financieros, aunque sea más fácil que el requerimiento original, también es un sobrecosto y no genera utilidad informativa. 
La Unión Europea, en su Directiva 2013/34/UE (Parlamento Europeo, 2013) estableció que, para las pymes europeas, la Norma Internacional podría resultar costosa, generado una sobrecarga de procesos para los objetivos y recursos de estas empresas. Por lo tanto, la NIIF de pymes no es obligatoria en la Unión Europea, como tampoco en algunos países de la región latinoamericana, como México o Argentina.

\subsection{Crítica a la definición de PYME}

Resulta al menos discutible el concepto de PYME que asume la NIIF. “Las pequeñas y medianas entidades son entidades que: a) no tiene obligación pública de rendir cuentas; y b) publican estados financieros con propósito de información general para usuarios externos" (IASB, 2016; NIIF PYMES, párrafo 1.2).

Definir una empresa por su obligación de rendir cuentas es tan inconveniente como suponer que las PYMES no tienen la obligación de rendir cuentas públicas. Esta posición inconveniente se debe, según nuestro punto de vista, a que se parte del supuesto según el cual el público está solo conformado por inversores financieros (Williams, 2006). En primer lugar, no se puede caracterizar una PYME por la información que debe producir. Especialmente cuando lo que se quiere definir en la norma es qué empresas deben aplicar el estándar que se ha producido y, en ciertos contextos, exigido como obligatoria. Tal situación se puede asimilar a definir un vehículo automotor por la persona que lo conduce: el conductor no dice nada de las características intrínsecas del vehículo. En segundo lugar, que la rendición de cuentas no se realice para inversores financieros no significa que las empresas no cotizadas no rindan cuentas ante terceros, la sociedad o el Estado (Pratten, 1998; Collis y Jarvis, 2002; Sunder, 2005; Williams y Ravenscroft, 2015). En síntesis, en relación con estos dos puntos, suponer que la contabilidad solo tiene razón de ser cuando es usada por los inversores, es un exabrupto y un desconocimiento histórico de la evolución y aporte de la contabilidad a la sociedad (Young, 2006).

Adicionalmente, es crítica la denominación de "pequeñas y medianas entidades" en lugar del nombre propio de "empresa". Este enfoque normativo está desarrollado para empresas con fines lucrativos e, incluso, con vocación bursátil. La denominación de "entidades" que adoptó la norma ha implicado que, por ejemplo, en Colombia se esté exigiendo a entidades sin fines lucrativos y a propiedades comunitarias (propiedades horizontales o edificios de domicilio) que produzcan su información financiera siguiendo esta NIIF. 


\subsection{Crítica por la visión restringida de la toma de decisiones}

La teoría de la decisión en administración, psicología, economía comportamental y ciencias sociales en general, ha presentado una evolución sustantiva (Simon, 1955; Kahneman, 2012; Williams y Ravenscroft, 2015). La visión de la toma de decisiones en los mercados financieros, según la cual las decisiones económicas se limitan a comprar o vender títulos de propiedad o de deuda de una empresa, puede denominarse en sentido estricto toma de decisiones de asignación de recursos en mercados (Staubus, 1961). Esta mirada no reconoce adecuadamente el rol integral de la contabilidad, incluso financiera, en la toma de decisiones dentro de la empresa. No todas las decisiones internas de la firma se basan en contabilidad de costos o gestión. La evolución histórica de la contabilidad mercantil nos muestra que la contabilidad es una conjugación de la información necesaria para gestionar la empresa, así como de la información para rendir cuentas y controlar el desempeño de la gerencia (Sunder, 2002). El control es una función sustantiva e intrínseca de la contabilidad y es la base para la correcta toma de decisiones (Williams y Ravenscroft, 2015; Watts y Zou, 2016). La contabilidad de gestión o estratégica, como un sistema independiente, es una realidad para empresas grandes. Pero las empresas pequeñas toman decisiones de asignación de recursos, por fuera de la bolsa, donde la contabilidad juega un rol importante.

El IASB señala en los fundamentos de conclusiones de la NIIF de PYMES, y en otros documentos institucionales, que "Los propietarios que son administradores utilizan los estados financieros para muchos propósitos. Sin embargo, la NIIF para las PYMES no tiene por objetivo el suministro de información a los propietarios para ayudarles a tomar decisiones de gestión". Este es, ciertamente, un sesgo antieconómico y expresa cierta incomprensión del contexto en el que operan las MIPYMES.

\subsection{Re-conceptualizar la noción de inversor}

Un requisito en la clarificación del proceso de toma de decisiones y del rol de la contabilidad como soporte para ello, implica redefinir el concepto de inversor. La noción convencional de inversor debe ser revaluada a la luz de los cambios socioeconómicos que se han planteado previamente. Las diferenciaciones que provienen de los vocablos en inglés pueden ayudarnos en tal distinción. Así, parece conveniente diferenciar entre inversor-bursátil (stockholder), inversor-propietario (shareholder), empresario-propietario (owner) y emprendedor (enterpreneur). 
El inversor bursátil está preocupado primordialmente por el rendimiento financiero, haciendo abstracción del proceso productivo; su lógica es la ampliación del valor financiero de los títulos que detenta, los cuales busca convertir en medios líquidos con celeridad. El inversor-propietario es aquel detentador de títulos de propiedad de una empresa concreta (accionista), por evaluación, preferencia o estrategia sobre una actividad económica específica; normalmente mantiene en el tiempo su posición de accionista en la empresa concreta. El empresario-propietario, es aquel dueño de una entidad, comprometido con ella por experticia, tradición y estrategia de negocio y que está vinculado a la gestión directa de la actividad económica y productiva. Finalmente, el emprendedor, es aquel sujeto que inicia una actividad productiva con vocación económica y de oportunidad (es decir no necesariamente para la subsistencia) y que busca socios inversores para llevar a cabo un modelo de negocio o una actividad que ha iniciado; normalmente su participación en la propiedad se materializa con su aporte industrial (conocimiento de la actividad o trabajo) o en especie. La cobertura de la categoría producción se refiere a bienes y servicios. Las necesidades de información para cada tipo de inversores son diferenciables porque sus procesos de decisión son diferentes. La clarificación de sus necesidades implica un programa de investigación de mediana envergadura, en el que está trabajando el autor del presente documento, entre otros académicos a nivel internacional.

Lo que podemos concluir preliminarmente es que el concepto de inversor no es unilateral ni universal, sino que, por el contrario, es fruto de una construcción contextual e histórica (Young, 2006). En el ámbito de las MIPYMES, quizás prevalecen los emprendedores y empresarios-propietarios. Por su parte, los principales inversores bursátiles, en la fase de financiarización contemporánea, son los inversores institucionales, aunque no los únicos.

\subsection{Tratamientos contables asimétricos en la NIIF de PYMES}

Una evaluación muy general de la NIIF para PYMES permite identificar una serie de falencias o tratamientos asimétricos en los requerimientos de dicha norma. Los temas más significativos son la imposibilidad de capitalización de los costos financieros, la restricción al reconocimiento de activos fruto de los procesos de investigación y desarrollo, y la exigencia de tratamientos contables de igual complejidad que los requeridos por las NIIF plenas, con poco sentido operativo y poco referente y utilidad práctica en la información para las pequeñas y medianas empresas.

Quizás el endeudamiento bancario, o con otros prestamistas, sea la fuente de financiación por excelencia para las micro empresas y para las PYMES. Mientras que los costos 
financieros en la adquisición, construcción o desarrollo de activos de Propiedad, Planta y Equipo de las grandes empresas o cotizadas pueden ser reconocidos como mayor valor del activo, cuando este sea apto (según la NIC 23, IASB, 2015b), la NIIF de PYMES no permite tal tratamiento. Tales desembolsos deberán ser reconocidos como gasto del periodo en las PYMES (Sección 25, NIIF de PYMES; IASB, 2016). Este tratamiento no permite diferir en el tiempo los efectos de esa financiación (por la vía de la depreciación), impactando en el corto plazo de manera negativa en el resultado de estas empresas.

Asimismo, la NIIF de PYMES no permite la capitalización de los desembolsos en la generación interna de intangibles. De esta manera, los desembolsos en las etapas de investigación y desarrollo deberán ser reconocidos como gastos (sección 18, NIIF de PYMES; IASB, 2016). Por su parte, en la NIC 38-Intangibles (IASB, 2015b) se permite a las empresas cotizadas, o que siguen las NIIF plenas, que bajo las condiciones establecidas activen los desembolsos realizados en la etapa de desarrollo. De esta manera, se proscriben las actividades de investigación y desarrollo en las PYMES, lanzándolas a la simple copia, quedando relegadas frente a las grandes empresas, al obstaculizar las actividades de generación de conocimiento e innovación, por los efectos financieros que implicaría emprender dichos procesos (tratar todos los desembolsos como gastos, periodo a periodo).

Se señala que estos tratamientos no son permitidos para las pequeñas y medianas entidades, porque implican un significativo grado de complejidad técnica. Paradójicamente, se obliga a las PYMES a realizar el deterioro del valor de los activos, estimando el importe recuperable de los mismos (Sección 27, NIIF de PYMES, IASB, 2016). La medición del valor razonable menos los costos de disposición y del valor de uso, para establecer el importe recuperable, implica una complejidad y un costo de información, seguramente más altos que los tratamientos anteriormente señalados. Pero lo más crítico es la falta de sentido y referente operativo en este tratamiento contable, lo que implica un complejo proceso para determinar hipotéticamente la Unidad Generadora de Efectivo. Este tratamiento carece de antecedente en las prácticas contables de las PYMES y, quizás, de muchas grandes empresas.

El efecto de estos tratamientos contables para la representación económico-financiera de la realidad impacta, por tanto, la eficiencia de las PYMES, generando asimetrías y problemas de competitividad frente a las grandes empresas cotizadas. 


\subsection{Retos para la relación entre forma organizacional y contabilidad}

Para finalizar este apartado, con base en Sunder (2005) y siguiendo el esquema de Churchill y Lewis (1983), se plantea que la contabilidad evoluciona y guarda una relación directa con la forma y el estado de desarrollo de las organizaciones.

En el caso de un proyecto empresarial naciente, los emprendimientos, la contabilidad requerida se asocia con la teneduría de libros. Allí la lógica de la formalización empresarial es la prioridad, buscando al mismo tiempo incorporar la racionalidad económica básica (los ingresos deben ser superiores a los costos, según la racionalidad instrumental del capitalismo), posibilitada por el registro y el control sistemático y oportuno de las transacciones. Asimismo, esto permite responder a los requerimientos mercantiles elementales para el surgimiento de la persona jurídica (para que la empresa se formalice, los sistemas legales de múltiples jurisdicciones exigen libros básicos de contabilidad y comercio). Por supuesto, hoy los libros son mayoritariamente electrónicos, dependiendo de las exigencias legales.

Cuando la empresa ha superado la etapa de su formalización y busca fortalecer su proceso productivo (de comercialización, manufactura o de prestación de servicios) requiere complementar sus libros contables con información detallada y precisa de inventarios y de costos. De esta manera requiere tasar del precio de venta y establecer el margen de utilidad para incrementar la racionalidad económica (buscando la eficiencia o el incremento del beneficio empresarial). Muchos sistemas contables en el mundo denominan a este enfoque "contabilidad analítica", una mezcla entre contabilidad comercial y de costos (Urquía, Pérez y Muñoz, 2011). Esta es la base de información para promover el primer peldaño hacia la productividad en la empresa, su objetivo es el control de la eficiencia.

En la evolución de la forma organizacional, una vez la eficiencia se va alcanzando, el emprendedor-propietario busca la asociación con otras personas naturales para aumentar la financiación, ampliar la actividad e incrementar los resultados operativos. Allíse necesita información que permita medir los resultados del proceso y conocer la situación económico-financiera de la empresa. Por ello surge la contabilidad financiera, sin necesidad de transitar a los procesos de evaluación de la misma por parte de terceros. Se busca informar a socios que no participan en la gestión directa, sobre el resultado empresarial: es la rendición de cuentas originaria o stewardship (Watts y Zuo, 2016). 
Posteriormente, con la empresa establecida, con un nicho de mercado claro, con un producto definido y con resultados económicos importantes, lo que se espera en el ciclo de negocios es el crecimiento o expansión. Allí la búsqueda de financiación ya no se concentra en la asociación con personas concretas, sino la búsqueda de capital o recursos frescos (muchas veces anónimos). Para conseguir financiación bancaria o en mercados de valores, se requiere que el Estado de Situación (Balance General), el Estado de Resultados, los Flujos de Efectivo y las Notas a los Estados Financieros (la Memoria), sean certificados por la administración y los expertos que los han preparado, así como dictaminados o asegurados por un tercero independiente. Allí surgen los informes financieros en plena propiedad (Sunder, 2005).

Por supuesto, esta información y su evolución guardarán mucha relación con las exigencias fiscales y tributarias de cada contexto. No obstante, hemos precisado la evolución de la contabilidad mercantil o financiera para remarcar la inconveniencia de requerir a una micro o a una pequeña empresa la preparación de reportes financieros como si fuera una gran empresa establecida que busca financiación bursátil. La teneduría de libros, la contabilidad analítica y la contabilidad financiera son mecanismos de control e información no necesariamente expuestos al público inversor, pero son básicos para la gestión y operación de la empresa. Es decir, son necesarios para la toma de decisiones internas de la empresa capitalista, asociados con la producción y el control de eficiencia.

Desde un punto de vista "glocal" es un reto reconocer esta situación. Dirigir efectivamente las prácticas contables desde normas o esquemas de regulación, implica comprender el contexto social y organizacional de las empresas que aplicarán dichas prescripciones; conviene, por tanto, pensar en tratamientos contables para las MIPYMES que reconozcan la realidad contextual en que actúan e impulsen la productividad organizacional, antes que el mero rendimiento financiero de corto plazo. Un único juego de normas globales, no es la solución para el caso de estas empresas. Menos cuando ese marco regulativo se dirige a otras prioridades, particularmente promoviendo la financiarización.

Exigir tratamientos para producir la información contable que no reconocen el contexto meso, macro y microeconómico, que no interpretan adecuadamente los procesos productivos de las empresas y que no identifican las necesidades de información concretas de los usuarios, llevará a que se conviertan en simples requerimientos formales, sin utilidad y sin aporte sustancial de la contabilidad a las empresas. La contabilidad así producida, podría ser vista como una sobrecarga y costo adicional para las MIPYMES (Parlamento Europeo, 2013). 


\section{Conclusiones}

Es clave entender el rol actual de la regulación contable financiera. Esto porque se presenta una hegemonía de los mercados financieros que promueve una financiarización económica con efectos complejos sobre la producción, el bienestar social y, sobre todo, en la distribución del valor. Hay una captación y concentración del valor por medio de las bolsas de valores.

Debemos entender que la toma de decisiones es un proceso muy diverso y complejo, que no sólo se refiere a la asignación de recursos en mercados financieros. La toma de decisiones en las organizaciones exige distinguir los diferentes tipos de inversores (Inversores-bursátiles, Inversores- propietarios, empresarios-propietarios y emprendedores), los contextos, así como las necesidades de información contable diferenciadas para diferentes usuarios. El énfasis de la información en las MIPYMES requiere que la toma de decisiones promueva la productividad y la eficiencia.

La contabilidad enfrenta diversas paradojas, entre ellas que la información contable debe servir tanto para el control (la rendición de cuentas) como para la toma de decisiones. El control no es simplemente la supervisión estatal, como tampoco la toma de decisiones es sólo decidir si comprar o vender títulos valores de propiedad o deuda en mercados financieros. Primero, la contabilidad debe servir para el control. Luego, cuando las organizaciones evolucionan y sus actores son responsables (Accountors), la contabilidad pasa a satisfacer otros usos. Surge así una utilidad ligada a la toma de decisiones para la productividad y la eficiencia operativa (impulsar el uso razonable y balanceado de recursos limitados, para conseguir fines u objetivos organizacionales). Este tipo de toma de decisiones es característico de las MIPYMES. La toma de decisiones en mercados financieros, enfocada en la maximización del valor de la inversión, es propia de operaciones bursátiles y es una realidad en la cima de la evolución organizacional (la gran empresa cotizada).

Es urgente reconocer que la contabilidad presta un mejor servicio a las organizaciones, a los procesos productivos y a la sociedad cuando entiende y representa el contexto local, sin perder de vista la dinámica global (por ello es indispensable un enfoque "glocal").

Estos retos ponen en evidencia la complejidad y las paradojas que enfrenta la contabilidad como práctica social e institucional. Esta complejidad y diversidad nos muestra la inconveniencia de un único juego de normas globales ante los múltiples problemas de una realidad económica y organizacional diversa. 
Este artículo se constituye en un marco teórico que puede impulsar y soportar investigaciones empíricas, que permitan caracterizar las necesidades de información de las MIPYMES, los efectos organizacionales y financieros de la aplicación de las NIIF y que permitan explicar y comprender los efectos de la transformación de las empresas y su dinámica de operación, en un contexto económico de financiarización. Esto reclama un programa de investigación de mediano alcance, posibilitado porque en países como Colombia se ha hecho obligatoria la aplicación de la NIIF-PYMES.

\section{Referencias}

Aglietta, M. y Reberioux, A. (2009). El capitalismo financiero a la deriva. El debate sobre el gobierno de la empresa. Bogotá: Universidad Externado de Colombia.

Albert, M. (1992). Capitalismo contra Capitalismo. Buenos Aires: Paidos, Estado y Sociedad.

Archel, P. y Gómez, M. (2014). Crisis de la valoración contable en el capitalismo cognitivo. Innovar, 52, 103-116.

Arnold, P. (2012). The political economy of financial harmonization: The East Asian financial crisis and the rise of international accounting standards. Accounting, Organization and Society, 37 (6), 361-381.

Bengtsson, E. (2011). Repoliticalization of accounting standard setting-The IASB, the EU and the global financial crisis. Critical Perspectives on Accounting, 22, 567-580.

Bezemer, D. (2010). Understanding financial crisis through accounting models. Accounting, Organization and Society, 35 (7), 676-688.

Biondi, Y. (2011). The pure logic of accounting: A critique of the fair value revolution. Accounting, Economics, and Law, 1 (1), Article 7.

Çelik, S., and Isaksson, M. (2014). Institutional investors and ownership engagement. OECD Journal: Financial Market Trends, Vol 2013/2, pp 93-114. https://www.oecd.org/corporate/Institutional-investors-ownership-engagement.pdf

Chua, W. F., and Taylor, S. L. (2008). The rise and rise of IFRS: An examination of IFRS diffusion. Journal of Accounting and Public Policy, 27, 462-473.

Churchill, N.C., and Lewis, V.L. (1983). The five stages of small business growth. Harvard Business Review, May-June, 30-50.

Collis, J., and Jarvis, R. (2002). Financial information and the management of small private companies. Journal of Small Business and Enterprise, 9, 2, 100-110.

Coriat, B., Coutrot, T., Pérez, R. y Weinstein, 0. (2012). Empresas: salir de la financiarización. En: Economistas aterrados. Cambiar de economía. Madrid: Fuhem-ecosocial, Libros de la catarata.

Deegan, C., and Unerman, J. (2011). Financial Accounting Theory, Second European Edition. McGraw Hill. 
Dufour, D. R. (2009). Gobernanza versus Gobierno. Cuadernos de Administración, 41, 27-37.

Eichengreen, B. (2000). Hacia una nueva arquitectura financiera internacional: propuesta después de la crisis asiática. México: 0xford University Press.

Giraldo, C. (2005). Financiarización: un nuevo orden social y político. En: Sistemas de Protección Social: entre la volatilidad económica y la vulnerabilidad social (pp. 233-283), t. 1. Bogotá: Facultad de Ciencias Económicas - CID, Universidad Nacional de Colombia.

Gruppi, L. (1978). El concepto de Hegemonía en Gramsci. México: Ediciones de Cultura Popular. Guttmann, R. (2009). Introducción al capitalismo conducido por las finanzas. En: Ola Financiera, 2, enero-abril, 20-56.

IASB (2010). Marco conceptual para la información financiera. London: International Financial Reporting Standards Foundation.

IASB (2015a). Norma Internacional de Información Financiera 13, Medición del Valor Razonable. London. International Financial Reporting Standards Foundation.

IASB (2015b). Normas Internacionales de Información Financiera. NIIF 2015. Libro Rojo. London. International Financial Reporting Standards Foundation.

IASB (2016). Norma Internacional de Información Financiera para las PYMES. Modificaciones 2015. London: International Financial Reporting Standards Foundation.

IASB (s.f.). Framework Project. Recuperado 13 de mayo de 2016, de: http://www.ifrs.org/Current-Projects/IASB-Projects/Conceptual-Framework/Pages/Conceptual-Framework-Summary.aspx

Investing.com. (s.f.). Tasas bancos centrales. Recuperado el 15 de mayo de 2016, de: http:// mx.investing.com/central-banks/

Jensen, M., and Meckling, W. (1976). Theory of the firm: Managerial behavior, agency costs and ownership structure. Journal of Financial Economics, 3 (4), 305-360.

Kahneman, D. (2012). Pensar rápido, pensar despacio. Barcelona: Debate.

Muller, J. (2014). An accounting revolution? The financialisation of standard setting. Critical Perspectives on Accounting, 25, 539-557.

OCDE y CEPAL (2013). Perspectivas económicas de América Latina 2013. Políticas de pymes para el cambio estructural. Organización para la Cooperación y el Desarrollo Económico Comisión Económica para América Latina y el Caribe. Santiago de Chile. http://dx.doi. org/10.1787/leo-2013-es

Orléan, A. (2006). El poder de las finanzas. Bogotá: Editorial Universidad Externado de Colombia. Parlamento Europeo. (2013). Directiva Europea 2013/34/UE, Contabilidad de Pymes. Recuperado 13 de mayo de 2016 de: http://eur-lex.europa.eu/legal-content/ES/TXT/PDF/?uri $=$ CELEX:32013L0034\&from=ES

Pérez, C. (2005). Technological revolutions and finance capital: The dynamics of bubbles and golden ages, Cheltenham: Edward Elgar. 
Power, M. (2010). Fair value accounting, financial economics and the transformation of reliability. Accounting and Business Research, 40 (3), 197-210.

Pratten, C. (1998). The Uses of Accounts of Small and Medium Sized Companies and the Effects of the Audit Exemption Regime. London: Institute of Chartered Accountants in England and Wales.

Robertson, R. (2003). Glocalización: tiempo-espacio y homogeneidad heterogeneidad. En: Monedero, J.C. Cansancio del Leviatán, problemas políticos en la mundialización (pp. 261-284). Madrid: Editorial Trotta.

Ryan, B., Scapens, R.W., Theobald, M. (2002). Research Method and Methodology in Finance and Accounting, (2a ed). Academic Press, Thomson Business Press.

Simon, H. A. (1955). A behavioral model for rational choice. Quarterly Journal of Economics, 69 (1), 99-118.

Staubus, G. (1961). A Theory of Accounting to Investors (pp. 53-71). Berkeley and Los Angeles: University of California Press.

Sunder, S. (2002). Decision Making and control: An accounting duality. In: Demski, Fellingham, Ijiri, and Sunder. Some Thoughts on the Intellectual Foundations of Accounting. Accounting Horizons, 16 (2), 157-168.

Sunder, S. (2005). Teoría de la contabilidad y el control. Bogotá: Universidad Nacional de Colombia. Tirol, J. (2001). Corporate Gobernance. Econometrica, 69 (1), 1-35.

UNCTAD (2010). Integrating Developing Countries SMEs into Global Value Chains. Geneva, Switzerland. United Nations Conference on Trade and Development.

Urquía, E., Pérez, R., and Muñoz, C. (2011). The impact of Accounting Information Systems (AIS) on performance measures: empirical evidence in Spanish SMEs. The International Journal of Digital Accounting Research, 11, 25-43.

Vercellone, C. (2009). Crisis de la ley del valor y devenir renta de la ganancia. Apuntes sobre la crisis sistémica del capitalismo cognitivo. En: La Gran crisis de la economía global (pp. 63-98). Madrid: Traficantes de sueños.

Watts, R., and Zuo, L. (2016). Understanding practice and institutions: A historical perspective. accounting horizons, 30 (3), 409-423.

Williams, P. (2006). Accounting for Economic Reality: Whose Reality, Which Justice? Accounting and the public interest, 6, 37-44.

Williams, P., and Ravenscroft, S. (2015). Rethinking decision usefulness. Contemporary Accounting Research, 32 (2), 763-788.

World Bank, and Dalberg Group (2011). Reporton Support to SMEs in Developing Countries Through Financial Intermediaries. Geneva.

Young, J. (2006). Making up users. Accounting, Organizations and Society, 31, 579-600. 